\title{
Humanzentrierte Künstliche Intelligenz: Erklärendes interaktives maschinelles Lernen für Effizienzsteigerung von Parametrieraufgaben
}

\author{
Christian Wirth ${ }^{1}$, Ute Schmid ${ }^{2(\bowtie)}$, und Stefan Voget $^{3}$ \\ 1 Continental Teves AG \& Co. OHG, Frankfurt, Deutschland \\ christian.2.wirth@continental-corporation.de \\ ${ }^{2}$ Kognitive Systeme, Universität Bamberg und Fraunhofer IIS Projektgruppe \\ Comprehensible AI, Bamberg, Deutschland \\ ute.schmid@uni-bamberg.de \\ 3 Continental Automotive GmbH, Regensburg, Deutschland \\ stefan.voget@continental-corporation.de
}

\begin{abstract}
Zusammenfassung. Verfahren der Künstlichen Intelligenz, insbesondere datenintensive Methoden des maschinellen Lernens, halten immer mehr Einzug in industrielle Anwendungen. Im Normalfall werden KIAnwendungen meist als fertige Black-Box-Komponenten betrachtet, welche nicht in der Lage sind, mit Anwendern zu interagieren. Am Beispiel von Parametrieraufgaben werden wir die dadurch entstehenden Probleme beleuchten und aufzeigen, wie Anwender eingebunden werden können. Für erfolgreiche Interaktion mit menschlichen Anwendern ist es notwendig, deren besonderen Erwartungen und Limitierungen explizit zu berücksichtigen. Neue, menschzentrierte Algorithmen, deren Interaktionsschnittelle auf psychologischen Erkenntnissen basiert, müssen entwickelt und identifiziert werden.
\end{abstract}

Schlüsselwörter: Humanzentrierte Künstliche Intelligenz • Erklärbare KI · Interaktives maschinelles Lernen

\section{$1 \quad$ Einleitung}

Künstliche Intelligenz (KI) ist eine Basistechnologie für viele neue Anwendungen und ist im modernen Leben allgegenwärtig: Im privaten Bereich zeigt Siri auf Zuruf den Weg, und Alexa ordert die Pizza im Restaurant um die Ecke. Ärzte

Die in diesem Beitrag berichtete Forschung wird unterstützt vom BMBFVerbundprojekt hKI-Chemie: Humanzentrierte KI in der chemischen Industrie, FKZ 01|S21023D, FKZ 01|S21023G.

(C) Der/die Autor(en) 2022

E. A. Hartmann (Hrsg.): Digitalisierung souverän gestalten II, S. 80-92, 2022. https://doi.org/10.1007/978-3-662-64408-9_7 
können Röntgenbilder mit KI exakter auswerten, und in Unternehmen entlasten Chatbots die Mitarbeiter im Service bei Kundenanfragen. In der Fertigung überwacht KI die Produktionsstrecke und sorgt für die Qualitätssicherung.

Um das enorme Potential von KI auszuschöpfen, müssen wir uns jedoch ihrer weitreichenden sozialen, wirtschaftlichen, rechtlichen und politischen Implikationen ebenso bewusst sein wie ihrer Auswirkungen auf unsere Moralvorstellungen, unsere gesellschaftlichen Normen und Werte. Den Kern der Verantwortungsethik für KI bildet ein nutzer- und mitarbeiterzentriertes Denken und Handeln auf allen Ebenen. KI als neues Werkzeug soll den Menschen unterstützen und ihm dienen (Ai et al, 2021). Häufig besteht Skepsis gegenüber KI. In der Praxis schwindet diese Skepsis jedoch schnell, weil die praktische Erfahrung und Umgang mit KI ihren Charakter als hilfreiches Instrument aufzeigt. Zwar kann KI durch die Lernfähigkeit das Wissen von vielen Mitarbeitern allgemein nutzbar machen. Es ist aber ein Missverständnis, daraus abzuleiten, dass der Mensch dadurch ersetzt würde. Vielmehr dient die KI denselben Menschen als ein neuartiges Werkzeug, um ihnen die eigentliche, kreative Arbeit zu erleichtern und repetitive Aufgaben zu reduzieren.

Hierbei ist $\mathrm{zu}$ beachten, dass sich diese Vision nur realisieren lässt, wenn die KI-Verfahren auch explizit auf Interaktionen ausgelegt sind. Die meisten, gängigen KI-Verfahren, etwa im Bereich Objekterkennung, unterliegen aber unrealistischen Anforderungen. Beispielsweise wird angenommen, dass es eine große Menge an Eingabedaten gibt, die bereits mit den erwarteten Ausgabewerten annotiert worden sind, und zudem ist im Regelfall keine Adaption an neue Daten oder Nutzer vorgesehen. Am Beispiel von Parameterisierungsaufgaben (Abschn. 2) werden wir diese Probleme genauer beleuchten (Abschn.3) und einen psychologischen Kontext (Abschn.4) setzen. Die zu berücksichtigenden Probleme lassen sich grob in zwei Kategorien gliedern: die Plausibiliserung der Ausgaben gegenüber dem Nutzer (Abschn.5) und den interaktiven Nutzen von menschlichen Eingaben (Abschn.6). Zum Abschluss geben wir einen Ausblick über aktuelle und zukünftige Arbeiten zur Entwicklung einer humanzentrierten KI (Abschn. 7).

\section{Use Case Parametrierung und Kalibrierung}

Im Automotivebereich werden häufig komplexe Anwendungen über Parametrierung und Kalibrierung den Bedingungen am endgültigen Einsatzort angepasst. Der Einsatzort kann beispielsweise eine Produktionsanlage oder das Fahrzeug sein. Die Parameter können zum Beispiel bestimmen, mit welchem Druck oder Temperatur eine Produktionsanlage zu betreiben ist oder wie sich ein Produkt unter unterschiedlichen Umweltbedingungen verhalten sollte. Die Suche nach den optimalen Parameterwerten ist oft ein zeitaufwendiger und teurer Vorgang. Häufig werden die Parametrierungs- und Kalibrierungsaufgaben unter engen Zeit- und Ressourcenvorgaben durchgeführt. Der manuelle Aufwand für die Parametrierung ist zunehmend schwer leistbar. 
Betrachten wir beispielsweise die Parametrierung von motorbetriebenen Schließsystemen in Fahrzeugen: Dabei handelt es sich um erstaunlich komplexe Systeme mit mehr als 20 Parametern, die eingestellt werden müssen, damit Schiebedach oder Kofferraum unter verschiedenen Umständen zuverlässig schließen. Hierbei berücksichtigen die Systeme Parameter wie Temperaturbereich, Neigungswinkel des Fahrzeugs, Transport- oder Schneelast. Gleichzeitig müssen Hindernisse, wie menschliche Körperteile (Kopf, Finger, etc.), indirekt und zuverlässig allein aus den Positions- und Motorparametern erkannt werden, da keine direkte Kraftmessung zur Verfügung steht. Der Kofferraum muss schließen, während die Quetschkraft im Falle eines Hindernisses so gering wie möglich sein sollte.

Eine solche Einklemmschutz-Funktionalität wird nicht nur für elektrische Kofferraumschließsysteme benötigt, sondern auch für andere Funktionen wie elektrische Fensterheber, Sitzsteuerung oder auch Schiebedächer.

Ohne KI-basierte Verfahren führen Experten mehrere Tests und Analysen am realen System durch. Das ist sowohl sehr zeitaufwendig als auch kostspielig. Allerdings basiert der manuell betriebene Ansatz auf langjähriger Erfahrung des Domänenexperten. Häufig haben diese Experten die Parameter ,im Gefühl‘, das heißt, sie wissen, welche Kombinationen funktionieren, können es aber nicht formell beschreiben. Zudem spiegeln die endgültigen Parametersätze die Erfahrungen und Vorlieben des Ingenieurs wieder, was zu unterschiedlicher Qualität und unterschiedlichem Verhalten des kalibrierten Systems führt. Eine direkte Implementierung in Software ist daher äußerst schwer.

Um solche Aufgaben mit Hilfe von Verfahren der KI zu lösen, sind verschiedene Aspekte zu berücksichtigen (Abb.1). Zum einen handelt es sich um so genannte Black-Box-Systeme, deren genaues Verhalten nicht mathematisch modelliert werden kann. Zudem sind nicht ausreichend viele, präzise Daten vorhanden, um klassische Lernverfahren anzuwenden. Dies bezieht sich sowohl auf die Eingabedaten als auch auf die Bewertung der Ergebnisse. Aufgrund dessen müssen spezielle Verfahren verwendet werden, die in der Lage sind mit menschlichen Bewertungen umzugehen, deren spezielle Eigenschaften zu berücksichtigen und auch mit kleinen Datenmengen zu arbeiten.

\section{Humanzentrierte KI für Parametrierung}

Ziel ist es, einen KI-basierten, kooperativen Ansatz für unsere Domänenexperten zu entwickeln. Wann immer möglich, werden die Parameter automatisch ausgewertet und nur bei Bedarf manuell durch den Experten gesetzt. Durch diesen manuellen Eingriff geben die Experten der KI Feedback, sodass sich die KI in weiteren Lernschleifen verbessern kann. Ziel ist es also nicht, den Domänenexperten durch eine voll automatisiert laufende KI zu ersetzen, sondern über einen interaktiven Ansatz den Domänenexperten zu entlasten, den Prozess zu beschleunigen und durch die KI zu neuen Parameterkombinationen zu kommen.

Continental hat ein Framework für die Parametrierung und Kalibrierung mit KI entwickelt, um diese Probleme zu lösen. Zunächst lernt die Software 
den ,Konsens“ über das gewünschte Verhalten von Autos, die bereits in der Vergangenheit kalibriert wurden. Eine dadurch gelernte Kostenfunktion stellt eine gleichbleibende Qualität aller Parametrierungen sicher, während einzelne Komponenten der Kostenfunktion leicht abgestimmt werden können, um die Präferenzen der Kunden oder Domänenexperten einzuarbeiten.
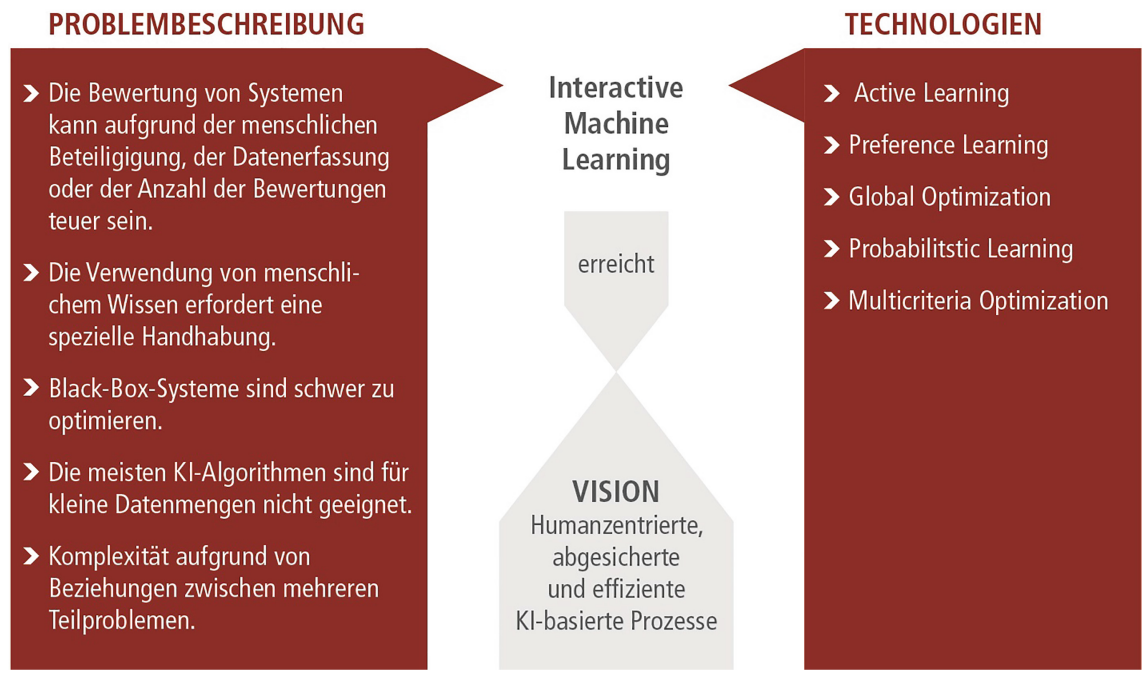

Abb. 1. Einordnung interaktiver KI

Daraufhin ermittelt das System auf der Grundlage erster grober Messungen automatisch einen optimierten Parametersatz. Am Ende erklärt das System dem Kalibrierungsingenieur die getroffenen Auswahlen. Ein unerfahrener Ingenieur kann im Allgemeinen den vom System gefundenen optimalen Parametersatz übernehmen, während ein erfahrener Kalibrierungsingenieur andere Optionen prüfen und diese von Fall zu Fall auswählen kann. Somit nimmt das System dem Menschen nicht die Kontrolle weg, sondern unterstützt einen humanzentrierten Entwicklungsansatz, basierend auf KI-Technologie.

Ist die Parametrisierung mithilfe der KI durchgeführt, ist es notwendig, die Entscheidung des Systems nachvollziehbar zu machen. Nur dann ist es für das menschliche Gegenüber möglich, gezielt mit dem System zu kooperieren und und wenn notwendig korrigierend einzugreifen. Nachvollziehbarkeit ist ebenfalls Voraussetzung für die Akzeptanz von KI-gestützten Verfahren. Dies gilt für alle Stakeholder, von den Ingenieuren, die das KI basierte Werkzeug nutzen, bis zu den Kunden, die das durch die KI parametrisierte Produkt kaufen.

Für die Methoden bestehen in der Interaktion mit den Menschen also zwei Herausforderungen: 
1. Interaktion mit dem Domänenexperten, um sein Wissen mithilfe der KI abzubilden.

2. Erklärbarkeit des Ergebnisses für Ingenieure, Kunden und andere Beteiligte. Warum ist die Lösung so gut, wie sie ist?

\section{Psychologische Aspekte bei der Kooperation von Mensch und KI-System}

Um humanzentrierte KI-Systeme zu entwickeln, müssen entsprechende Ansätze für die Erklärungsgenerierung und die Interaktionsschnittstelle entwickelt werden. Zudem ist es unverzichtbar, psychologische Erkenntnisse bei der Gestaltung zu berücksichtigen (Miller, 2019). Einerseits müssen Spezifika der kognitiven Verarbeitung von Informationen beachtet werden, andererseits müssen Fragen des Vertrauens in technische Systeme und deren Akzeptanz adressiert werden.

Im Fokus des Use Cases steht die Interaktion eines KI-Systems mit menschlichen Experten in der Produktion. Damit ein effizienter und effektiver Informationsaustausch zwischen KI-System und Mensch umgesetzt werden kann, muss die Information so präsentiert und kommuniziert werden, dass

- die kognitive Belastung möglichst gering gehalten wird,

- die Information in geeigneter Modalität und Granularität präsentiert wird,

- unpräzise und unvollständige Informationseingaben vom System akzeptiert und geeignet verarbeitet werden können,

- das System robust und adaptiv gegenüber Veränderungen ist, die sich über die Zeit und durch Personenwechsel ergeben.

Kognitive Belastung entsteht einerseits durch die Komplexität der Aufgabenanforderung oder Information selbst (intrinsic cognitive load), andererseits durch die Art, in der die Information präsentiert wird (extrinsic cognitive load) (Sweller et al, 2011). Bei komplexen Aufgaben können Hierarchisierung und Segmentierung der Information helfen, die intrinsische Komplexität zu reduzieren. Zudem sollten nur relevante Aspekte der Information präsentiert werden, um sogenannte dilusion effects zu vermeiden (Miller, 2019). Auch die angemessene Wahl der Modalität, in der die Information präsentiert wird, hat einen Einfluss auf die kognitive Belastung. Abstrakte Informationen werden in der MenschMensch-Kommunikation üblicherweise sprachlich übermittelt, bei grundlegenden perzeptuellen, insbesondere visuellen Kategorien ist eine Informationsübermittlung in Form von bildhafter Information dagegen oft effizienter (Slough und McTigue, 2010). Visuelle Hinweisreize sind zudem hilfreich, um gezielt die Aufmerksamkeit auf bestimmte Information zu lenken (Chun, 2000).

Menschliche Informationsverbeitung ist zudem überwiegend qualitativ. So werden Unterschiede zwischen metrischen Größen oder Trends in Zeitreihen typischerweise qualitativ repräsentiert und verarbeitet. Entsprechende Modelle 
zum qualitativen Schließen werden sowohl im Bereich KI (Bobrow, 1984) als auch in der Kognitionspsychologie entwickelt (Johnson-Laird, 1980).

Die genannten Aspekte betreffen die Kommunikation vom System zum Menschen. Umgekehrt müssen menschzentrierte KI-Systeme so umgesetzt werden, dass sie robust gegenüber unpräzisen und unvollständigen Eingaben sind. Zudem muss mitbedacht werden, dass sich Urteile intrapersonell über die Zeit verschieben können und dass im größeren Kontext der Arbeitsorganisation zu erwarten ist, dass zu verschiedenen Zeitpunkten verschiedene Personen mit dem System interagieren. Hier ist zu erwarten, dass verschiedene Personen mehr oder minder unterschiedliche Beurteilungskriterien haben. Zudem können je nach Aufgabenkontext, aktueller Belastung und aufgrund persönlicher Unterschiede, verschiedene Arten der Informationspräsentation präferiert werden (Ellwart et al, 2019).

Die Berücksichtigung dieser Aspekte kann aber nur gewährleisten, dass eine interaktive Nutzung mit menschlichen Nutzern möglich ist. Dies ist aber nicht ausreichend, um auch Vertrauen und Akzeptanz zu schaffen. Solche Aspekte sind gerade im industriellen Umfeld von hoher Relevanz, da hier eine (ethische) Verantwortung gegenüber Kunden, Gesetzgebern und Mitarbeitern besteht. Letztere haben häufig die Befürchtung, dass sie durch autonome intelligente Systeme ersetzt werden könnten. In den meisten Kontexten ist jedoch davon auszugehen, dass die komplexen Prozesse nur durch geeignete Kooperation von Mensch und Maschine realisierbar sind. Entsprechend muss deutlich vermittelt werden, dass es teilweise zwar eine neue Aufgabenverteilung zwischen Mensch und Maschine geben wird, aber die menschliche Expertise unverzichtbar ist.

Forschung zur menschzentrierten KI adressiert die beschriebenen kognitiven und organisatorischen Anforderungen durch die Entwicklung entsprechender Methoden. Insbesondere werden Erklärbarkeit, Fairness und Validierbarkeit von KI-Systemen betrachtet. Der Fokus ist dabei auf die Umsetzung dieser Anforderungen für datenintensive Ansätze des tiefen Lernens gerichtet, da hier die gelernten Modelle selbst für die Entwickler nicht nachvollziehbar und nicht transparent sind.

Um Modelle korrigierbar zu machen, werden zunehmend interaktiv lernende Systeme entwickelt (Holzinger, 2016), bei denen Menschen die Ausgaben eines Systems korrigieren können. Die Korrekturen werden dann zur Adaptation des Systems genutzt. Um die Interaktion geeignet zu gestalten, bedarf es Ansätze, die die Systementscheidung sowie die für die Entscheidung genutzte Information transparent und nachvollziehbar machen. Diese Anforderung wird vom Forschungsgebiet Erklärbare KI (explainable AI, XAI) adressiert (Abschn.5). Zur Kommunikation mit Domänenexperten sind visuelle XAI-Ansätze häufig nicht aussagestark genug. Um komplexe Zusammenhänge zu kommunizieren sind häufig verbale oder andere Arten symbolischer Erklärungen notwendig (Schmid, 2021), im Zweifel unter Verwendung eines mehrschrittigen Dialogs (Finzel et al, 2021). Generell ist aber zu beachten, dass Erklärungen nicht zu komplex sein sollten, um die kognitive Belastung gering zu halten (Ai et al, 2021). 
Erklärungen sind ebenfalls relevant, um die Korrektheit und Fairness von gelernten Modellen überprüfbar zu machen. So konnte in einem psychologischen Experiment gezeigt werden, dass Erklärungen sowohl das Verständnis von Systementscheidungen als auch das Vertrauen in das System erhöhen können (Thaler und Schmid, 2021). Allerdings ist dabei zu gewährleisten, dass die generierten Erklärungen auch tatsächlich die Systementscheidungen abbilden, damit das Vertrauen ins System gerechtfertigt ist (Teso und Kersting, 2019).

\section{Methoden zur Erklärung von KI}

XAI hat viele Facetten (Abb. 2). Es kann in verschiedenen Situationen und auf verschiedene Arten genutzt werden (Miller, 2019; Barredo Arrieta et al, 2020; Vilone und Longo, 2020). Erklärbares maschinelles Lernen bietet das Potenzial, Stakeholdern Einblicke in das Modellverhalten zu geben, indem verschiedene Methoden verwendet werden.

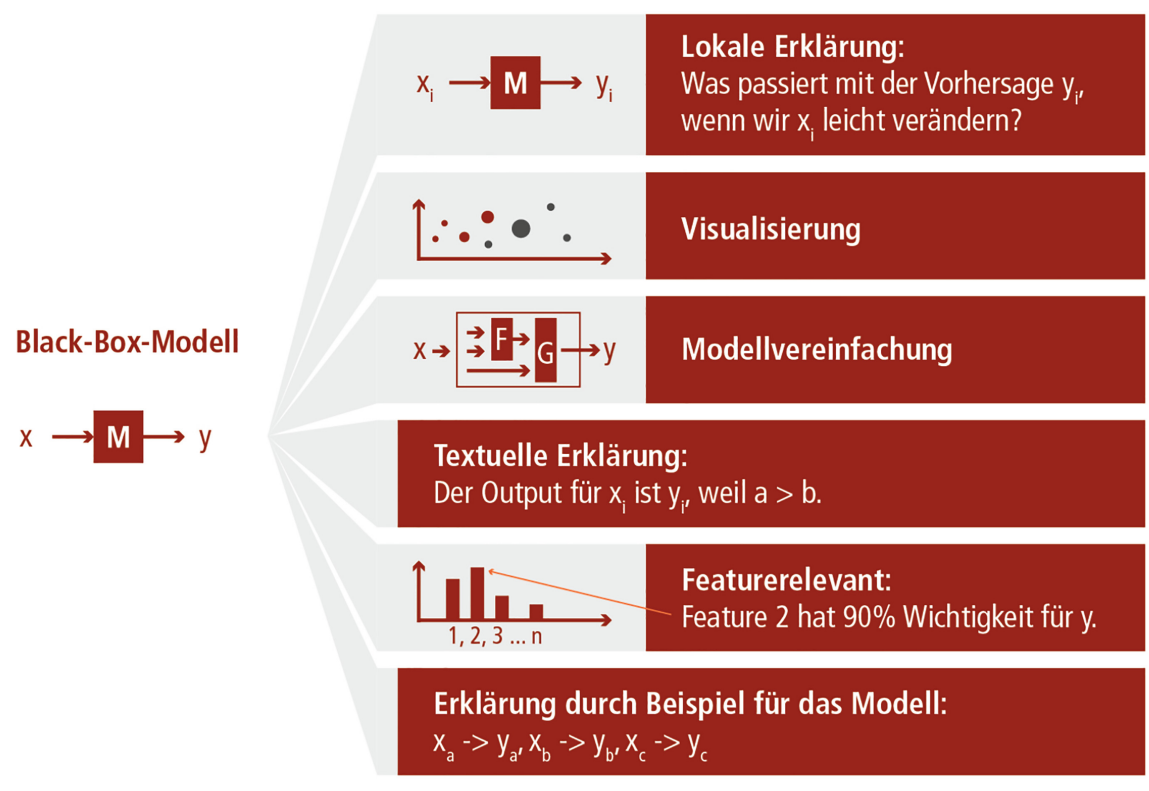

Abb. 2. Typen der Erklärung

Dennoch gibt es bisher noch wenig Verständnis darüber, wie Organisationen diese Methoden in der Praxis nutzen. Tab. 1 listet einige Beispiele, bei denen die XAI-Methoden Vorteile bringen können.

Einige Visualisierungswerkzeuge haben sich bereits in der kurzen Zeit, in der es sie gibt, als sehr beliebt erwiesen. Aber helfen sie auch? Obwohl viele Methoden vorgeschlagen wurden, gibt es relativ wenige experimentelle Studien, die 
Tab. 1. Nutzer einer Erklärung

\begin{tabular}{l|l}
\hline Für wen? & Warum? \\
\hline $\begin{array}{l}\text { Domänenexperte/-nutzer } \\
\text { (z. B. Arzt) }\end{array}$ & $\begin{array}{l}\text { Vertrauen in das Modell, wissenschaftliches Wis- } \\
\text { sen gewinnen }\end{array}$ \\
\hline Betroffene & $\begin{array}{l}\text { Verstehen ihrer Situation, Überprüfung auf faire } \\
\text { Entscheidungen }\end{array}$ \\
\hline $\begin{array}{l}\text { Aufsichtsbehörden und } \\
\text { Agenturen }\end{array}$ & $\begin{array}{l}\text { Bescheinigung der Gesetzeskonformität, } \\
\text { Durchführung von Audits }\end{array}$ \\
\hline $\begin{array}{l}\text { Datenwissenschaftler, } \\
\text { Entwickler }\end{array}$ & $\begin{array}{l}\text { Verbesserung der Produkteigenschaften, } \\
\text { Entwicklung neuer Funktionalitäten }\end{array}$ \\
\hline Produktverantwortliche & Gewährleistung der Produkteigenschaften \\
\hline $\begin{array}{l}\text { Manager und Vor- } \\
\text { standsmitglieder }\end{array}$ & $\begin{array}{l}\text { Beurteilung der Einhaltung von Vorschriften, KI- } \\
\text { Anwendungen im Unternehmen verstehen }\end{array}$ \\
\hline
\end{tabular}

untersucht haben, ob diese Modelle die beabsichtigten Effekte erzielen, wie z. B. Menschen dazu zu bringen, den Vorhersagen eines Modells genauer zu folgen, wenn es für sie von Vorteil ist, oder sie in die Lage zu versetzen, zu erkennen, wenn ein Modell einen Fehler gemacht hat.

$\mathrm{Zu}$ den Einschränkungen der Methoden gehört je nach Nutzerfall, dass man Domänenexperten zur Bewertung der Erklärungen benötigt. Es besteht das Risiko von falschen Korrelationen, die sich in Modellerklärungen widerspiegeln. Außerdem besteht häufig eine Latenz bei der Berechnung und Darstellung von Erklärungen in Echtzeit. In Altmann et al (2020) haben die Autoren mehrere Methoden untersucht und deren Limitierungen analysiert. Alle Methoden können verwendet werden, um das Verhalten und die Vorhersagen von trainierten Machine-Learning-Modellen zu erklären. Aber die Interpretationsmethoden funktionieren nur eingeschränkt wenn ein Modell Interaktionen modelliert, wenn Merkmale stark miteinander korrelieren, wenn das Modell kausale Zusammenhänge nicht korrekt modelliert oder wenn die Parameter der Interpretationsmethode nicht richtig gesetzt sind.

\section{Interaktive KI-Methoden}

Die grundlegende Idee interaktiver KI-Methoden ist die Integration des Menschen in KI-Verfahren um eine Kooperation und Adaption zu ermöglichen. Hierbei wird der im Regelfall statische Prozess des Sammelns, Trainierens und Anwendens von Daten durch einen iterativen Zyklus ersetzt (Abb. 3). Das heißt, dass der Anwender iterativ neue Daten erzeugen kann, die das gelernte Modell verändern oder verbessern. Hierbei müssen die in Abschn. 4 genannten Aspekte berücksichtigt werden, wobei die in Abb. 4 genannten Eigenschaften des interaktiven Ansatzes genutzt werden können. 


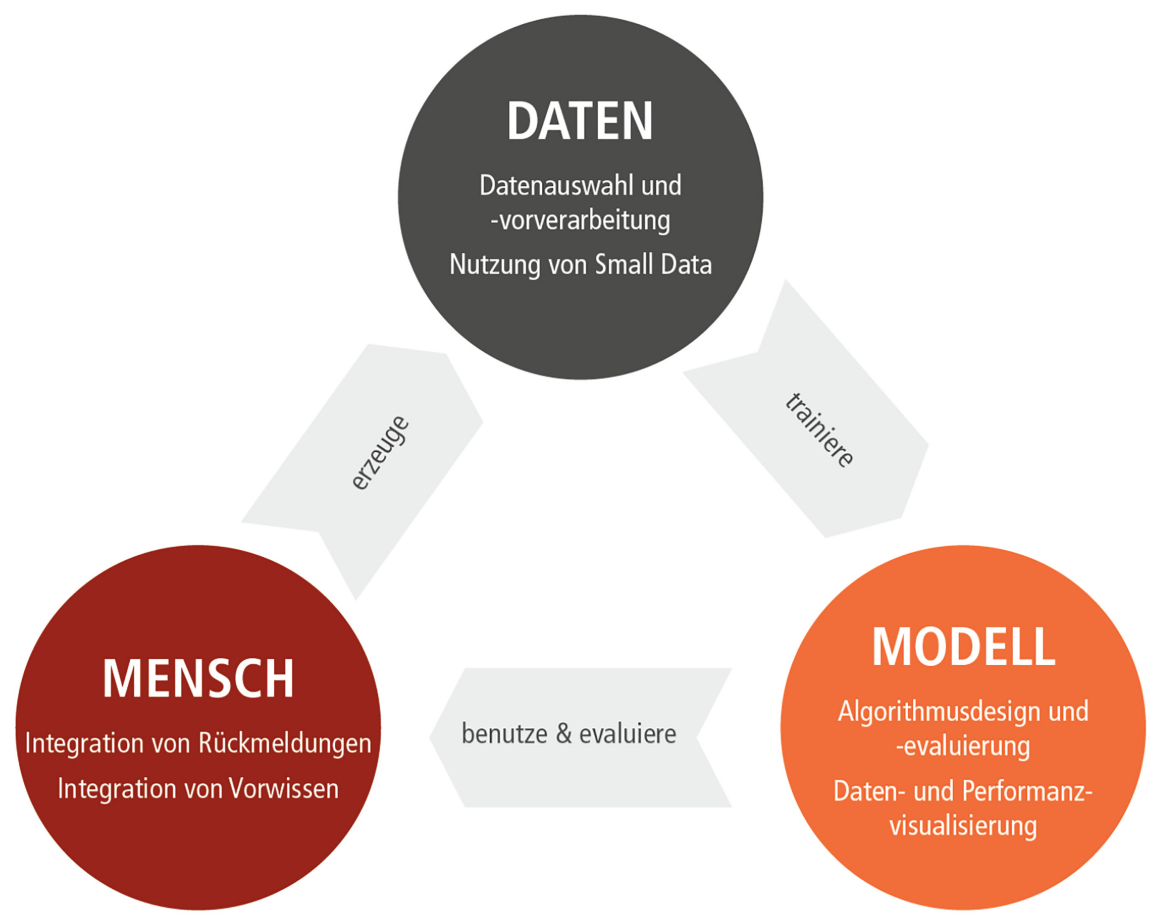

Abb. 3. Interaktiver KI-Zyklus

\subsection{Kognitive Belastung}

Um die kognitive Belastung (Abschn.4) des Anwenders zu reduzieren, kann aktives Lernen (Settles, 2012) genutzt werden, womit die Anzahl der gestellten Rückfragen minimiert wird. Klassisches aktives Lernen verwendet so genannte poolbasierte Ansätze, bei denen davon ausgegangen wird, dass ungelabelte Daten vorhanden sind und es ausreicht, eine Teilmenge für die Auswertung auszuwählen. Eine Alternative ist das aktive Lernen mit Zugehörigkeitsabfragen (Wang et al, 2015), bei dem angenommen wird, dass wir völlig neue Datenpunkte eines bekannten Datenraums anfordern können. Beispielsweise haben wir eine abstrakte Beschreibung von Bildern und können neue Bilder abfragen, indem wir eine neue abstrakte Beschreibung definieren. Im Allgemeinen sollte auch berücksichtigt werden, dass viele moderne Methoden des aktiven Lernens probabilistische Informationen verwenden (Kirsch et al, 2019) oder kalibrierte Konfidenzen annehmen (Wang et al, 2017), weshalb diese von fortschrittlichen Methoden des probabilistischen Lernens profitieren können.

Vor allem Ansätze mit Zugehörigkeitsabfragen sind für Parametrierungsaufgaben von Relevanz. Diese Verfahren erlauben es, Parametersätze zu bestimmen, auch wenn diese bisher noch nicht ausprobiert worden sind. 


\subsection{Qualitative Aussagen}

Da Menschen nur schwer in der Lage sind, quantitative Aussagen zu treffen (Abschn.4), ist es von Vorteil, qualitative Bewertungsschemata anzuwenden. Diese sind bereits in diversen Anwendungen zu finden, wie bei ordinalen Produktbewertungen oder impliziten, paarweise Präferenzaussagen (durch Anklicken) bei Suchmaschinen.
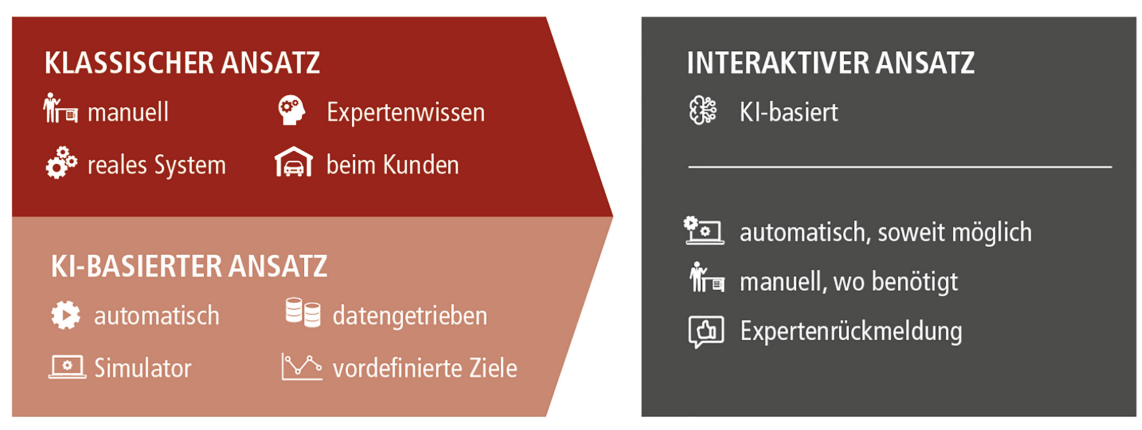

Abb. 4. Ansatz mit interaktiver KI

Allgemein lassen sich qualitative Aussagen als Präferenzen interpretieren, da auch eine ordinale Bewertung eine implizite, paarweisen Präferenz über Beispiele mit einem niedrigeren Rang darstellt. Auch regelbasierte Präferenzaussagen lassen sich in einen paarweisen Vergleich übertragen. Somit sind präferenzbasierte Lernmethoden (Fürnkranz und Hüllermeier, 2010) eine wichtige Verfahrensklasse für interaktive KI-Methoden. Zudem lassen sich diese Methoden auch für Probleme mit Entscheidungssequenzen nutzen (Wirth et al, 2017).

Für Parameterisierungsprobleme werden solche Methoden genutzt, um menschliches Wissen maschinell erfassen zu können. Hierbei können Domänenexperten bekannte oder neue Beispiele mit allgemeinen Regeln bewerten oder direkt untereinander vergleichen. Mit dieser Information kann eine Bewertungsfunktion abgeleitet werden, welche alle Aussagen so weit wie möglich erfüllt. Die hierdurch gelernte Bewertungsfunktion ermöglicht es, nun nicht nur automatisiert Bewertungen vorzunehmen, sondern auch das implizite „Gefühl“ (Abschn. 2) explizit darzustellen. Somit kann die gewonnene Information überprüft oder zum Anlernen neuer Mitarbeiter genutzt werden.

\subsection{Unpräzise Signale}

Wie in Abschn. 4 beschrieben, ist es für Menschen schwierig präzise Bewertungen vorzunehmen. Das heißt, auch die qualitativen Aussagen können nicht als komplett korrekt und präzise angenommen werden, sondern sollten als beste Schätzung angesehen werden. 
Daher sollten für interaktive Anwendungen keine deterministischen, sondern probabilistische Verfahren (Murphy, 2021) oder fuzzy reasoning (Wu, 2014) verwendet werden. Diese ermöglichen es, Schlüsse über die Korrektheit von Aussagen zu nutzen oder zu lernen. Somit ist es möglich, Modelle zu finden, welche alle Aussagen nur soweit wie möglich erfüllen.

Diese Eigenschaft ist auch nötig, um mit dem Problem der Veränderlichkeit (Abschn. 4) umzugehen. Solange anzunehmen ist, dass es ein stationäres Modell gibt, welches die menschlichen Aussagen adäquat abbilden kann, ist es wiederum ausreichend, alle Aussagen so weit wie möglich zu erfüllen. Das heißt, es müssen eventuell zusätzlich Datenpunkte erhoben werden (mithilfe des aktiven Lernens), aber es wird davon ausgegangen, dass die menschliche Bewertung im Durchschnitt keiner zeitlichen Veränderung unterliegt.

\subsection{Black-Box-Optimierung}

Bei vielen Anwendungsfällen ist zudem zu berücksichtigen, dass die Eigenschaften des zu optimierenden Modells nicht bekannt sind (Abschn. 2). Das heißt, es können nur begrenzt klassische Optimierungsverfahren wie Gradientenabstieg genutzt werden, da diese z. B. eine einzigartige, optimale Lösung annehmen. Somit ist es nötig, Verfahren der globalen Optimierung (Deb et al, 2002; Mockus, 2012) zu nutzen, welche wenig bis keine Annahmen über die Struktur des unterliegenden Problems benötigen. Üblich in solch einem Szenario ist die Anwendung von genetischen bzw. evolutionären Algorithmen (Deb et al, 2002), welche aber eine hohe Anzahl von Evaluierungen benötigen. Das heißt, in Fällen, bei denen die Evaluierung eines Datenpunktes menschliche Unterstützung benötigt, kommen die Probleme der kognitiven Belastung wieder zum Tragen (Abschn. 4).

Eine Alternative stellt Bayesian Optimization (Mockus, 2012) dar, welche deutlich effizienter ist, aber begrenzt in der Größe des verwendbaren Datenraums. Diese Verfahren kombinieren probabilistisches Lernen mit aktivem Lernen, um einen bekannten Lösungsraum nach einer guten Lösung zu durchsuchen.

\section{Ausblick}

Humanzentrierte KI ist von großer Bedeutung, gerade im industriellen Umfeld, da diese Methoden die Akzeptanz neuer KI-Ansätze verbessern können. Zudem ermöglichen Mensch-Maschine-Kooperationen neue Anwendungsfelder, die mit klassischen KI-Verfahren nicht erschlossen werden können. Um eine praxisorientierte Nutzung zu gewährleisten, müssen aber verschiedenste Perspektiven gemeinschaftlich berücksichtigt werden. Die psychologische, ethische und regulative Perspektive stellen unterschiedliche Anforderungen an die technische Seite, welche auf interaktiven und erklärbaren KI-Verfahren basiert. Zudem erzeugt die technologische Perspektive aber Annahmen und Limitierungen die wiederum psychologisch und ethisch betrachtet werden müssen. Daher sehen wir für die Zukunft einen Bedarf an domänenübergreifenden Entwicklungsprojekten, wie das vom BMBF geförderte Projekt, ,humanzentierte KI in der Chemischen 
Industrie" welches in 2021 gestartet ist und sich dem Ziel eines ganzheitlichen Lösungsansätzes widmet. Durch das Einbinden aller Interessengruppen kann durch solche Projekte bereits während der Entwicklung die Grundlage für die nötige Akzeptanz neuer KI-Methoden geschaffen werden.

\section{Literatur}

Ai, L., Muggleton, S.H., Hocquette, C., Gromowski, M., Schmid, U.: Beneficial and harmful explanatory machine learning. Mach. Learn. 110(4), 695-721 (2021)

Altmann, T., et al.: Limitations of interpretable machine learning methods. https:// compstat-lmu.github.io/iml_methods_limitations/ (2020)

Barredo Arrieta, A., et al.: Explainable artificial intelligence (XAI): concepts, taxonomies, opportunities and challenges toward responsible AI. Inf. Fusion 58, 82-115 (2020)

Bobrow, D.G.: Qualitative reasoning about physical systems: an introduction. Artif. Intell. 24(1-3), 1-5 (1984)

Chun, M.M.: Contextual cueing of visual attention. Trends Cognit. Sci. 4(5), 170-178 (2000)

Deb, K., et al.: A fast and elitist multiobjective genetic algorithm: NSGA-II. IEEE Trans. Evol. Comput. 6(2), 182-197 (2002)

Ellwart, T., et al.: Intentional forgetting in socio-digital work systems: system characteristics and user-related psychological consequences on emotion, cognition, and behavior. AIS Trans. Enterp. Syst. 4(1). https://www.aes-journal.com/index.php/ ais-tes/article/view/16 (2019)

Finzel, B., Taer, D., Scheele, S., Schmid, U.: Explanation as a process: user-centric construction of multi-level and multi-modal explanations. In: KI 2021: Advances in Artificial Intelligence-44th German Conference on AI, S. 80-94, Springer, Heidelberg. https://dblp.uni-trier.de/rec/conf/ki/FinzelTSS21.html?view=bibtex (2021)

Fürnkranz, J., Hüllermeier, E. (Hrsg.).: Preference Learning. Springer, Heidelberg (2010)

Holzinger, A.: Interactive machine learning (IML). Inform.-Spektrum 39(1), 64-68 (2016)

Johnson-Laird, P.N.: Mental models in cognitive science. Cognit. Sci. 4(1), 71-115 (1980)

Kirsch, A., et al.: Batchbald: efficient and diverse batch acquisition for deep Bayesian active learning. In: Advances in Neural Information Processing Systems 32: Annual Conference on Neural Information Processing Systems, S. 7024-7035 (2019)

Miller, T.: Explanation in artificial intelligence: insights from the social sciences. Artif. Intell. 267, 1-38 (2019)

Mockus, J.: Bayesian Approach to Global Optimization: Theory and Applications. Mathematics and its Applications. Springer, Netherlands (2012)

Murphy, K.P.: Probabilistic Machine Learning: An introduction. MIT Press, Cambridge, MA. https://probml.ai (2021)

Schmid, U.: Interactive learning with mutual explanations in relational domains. In: S. Muggleton and N. Chater (Hrsg.). Human-like Machine Intelligence, S. 337-353. Oxford University Press, Oxford (2021)

Settles, B.: Active Learning. Morgan \& Claypool Publishers, San Rafael, CA (2012)

Slough, S.W., McTigue, E.M.: Introduction to the integration of verbal and visual information in science texts. Read. Psychol. 31(3), 206-212 (2010) 
Sweller, J., Ayres, P., Kalyuga, S.: Cognitive Load Theory, Explorations in the Learning Sciences, Instructional Systems and Performance Technologies, Bd.1. Springer, Heidelberg (2011)

Teso, S., Kersting, K.: Explanatory interactive machine learning. In: Proceedings of the 2019 AAAI/ACM Conference on AI, Ethics, and Society, S. 239-245 (2019)

Thaler, A., Schmid, U.: Explaining machine learned relational concepts in visual domains effects of perceived accuracy on joint performance and trust. In: Proceedings of the 43rd Annual Meeting of the Cognitive Science Society, Cognitive Science Society, S. 1705-1711 (2021)

Vilone, G., Longo, L.: Explainable artificial intelligence: a systematic review. CoRR abs/2006.00093. https://arxiv.org/abs/2006.00093 (2020)

Wang, K., et al.: Cost-effective active learning for deep image classification. IEEE Trans. Circuits Syst. Video Technol. 27(12), 2591-2600 (2017)

Wang, L., et al.: Active learning via query synthesis and nearest neighbour search. Neurocomputing 147, 426-434 (2015)

Wirth, C., Akrour, R., Neumann, G., Fürnkranz, J.: A survey of preference-based reinforcement learning methods. J. Mach. Learn. Res. 18(136), 1-46 (2017)

$\mathrm{Wu}, \mathrm{Q} .:$ Knowledge granulation, rough entropy and uncertainty measure in incomplete fuzzy information system. Comput. Inform. 33(3), 633-651 (2014)

Open Acces Dieses Kapitel wird unter der Creative Commons Namensnennung 4.0 International Lizenz (http://creativecommons.org/licenses/by/4.0/deed.de) veröffentlicht, welche die Nutzung, Vervielfältigung, Bearbeitung, Verbreitung und Wiedergabe in jeglichem Medium und Format erlaubt, sofern Sie den/die ursprünglichen Autor(en) und die Quelle ordnungsgemäßnennen, einen Link zur Creative Commons Lizenz beifügen und angeben, ob Änderungen vorgenommen wurden.

Die in diesem Kapitel enthaltenen Bilder und sonstiges Drittmaterial unterliegen ebenfalls der genannten Creative Commons Lizenz, sofern sich aus der Abbildungslegende nichts anderes ergibt. Sofern das betreffende Material nicht unter der genannten Creative Commons Lizenz steht und die betreffende Handlung nicht nach gesetzlichen Vorschriften erlaubt ist, ist für die oben aufgeführten Weiterverwendungen des Materials die Einwilligung des jeweiligen Rechteinhabers einzuholen.

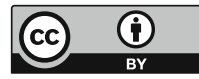

